\title{
X-ray BPM-Based Feedback System at the APS Storage Ring
}

\author{
O. Singh, L. Erwin, G. Decker, R. Laird and F. Lenkszus \\ Advanced Photon Source, Argonne National Laboratory, \\ 9700 South Cass Avenue, Argonne, IL 60439
}

\begin{abstract}
At the Advanced Photon Source (APS) storage ring, the X-ray beam position monitors (X-BPMs) measure accurate photon position down to the submicron level. This level of stable measurement has been possible due to 1) superior thermal insulation and vibration damping of the X-ray BPM support structure [1],2) minimal dependence on the bunch pattern and intensity variations, and 3 ) use of ultrastable preamplifiers and processing electronics. A new X-BPM interface is under development and will be discussed here. This interface will be integrated into the existing rf-based orbit feedback systems. To study preliminary results, an experimental X-BPM orbit feedback set-up was developed and implemented in one of the bending magnet beamlines. The results from this set-up are encouraging. For an operational fill, a typical orbit drift of 30 microns (at X-ray BPMs) has been reduced to less than 5 microns. The fill-to-fill photon orbit reproducibility has been improved from 75 microns to less than 10 microns.
\end{abstract}

\section{INTRODUCTION}

Orbit feedbacks, based on rf BPMs, are routinely used at the Advanced Photon Source (APS) storage ring and other facilities. The superior performance of the feedback systems at APS has provided the desired orbit stability to users, although the orbit stability requirements are becoming tighter as the beam size gets smaller. At the APS storage ring, with a current vertical beam size of 18 microns at $1 \%$ coupling, a $5 \%$ orbit stability requirement would be less than 1 micron, which has been difficult to achieve. The rf-BPM drifts due to intensity dependence, the mechanical and thermal effects, and the bunch pattern dependence are some of the limitations. Since X-ray BPMs are known to be much less dependent on the above-mentioned factors, there has been a shift towards integrating X-ray BPMs into orbit feedback systems.

The workstation-based orbit correction system [2] requires data collection at 10 $\mathrm{Hz}$, whereas the digital signal processor (DSP)-based orbit correction system (realtime feedback) [3] 'requires data collection up to $2 \mathrm{kHz}$. The present data collection rate is set at $1.6 \mathrm{kHz}$. A new X-BPM interface, which will meet the above data rate and other requirements, is under development. A brief design description of this system is provided in this paper. 


\section{DISCLAIMER}

This report was prepared as an account of work sponsored by an agency of the United States Government. Neither the United States Government nor any agency thereof, nor any of their employees, make any warranty, express or implied, or assumes any legal liability or responsibility for the accuracy, completeness, or usefulness of any information, apparatus, product, or process disclosed, or represents that its use would not infringe privately owned rights. Reference herein to any specific commercial product, process, or service by trade name, trademark, manufacturer, or otherwise does not necessarily constitute or imply its endorsement, recommendation, or favoring by the United States Government or any agency thereof. The views and opinions of authors expressed herein do not necessarily state or reflect those of the United States Government or any agency thereof. 


\section{DISCLAIMER}

Portions of this document may be illegible in electronic image products. Images are produced from the best available original document. 
There are two X-ray BPMs installed in each of the insertion device (ID) and bending magnet beamlines. Bending magnet X-ray BPMs provide vertical position information only, while insertion device.X-ray BPMs have both vertical and horizontal readbacks. Before the insertion device X-BPMs can be used for orbit feedback, the X-BPM blade's contamination due to background stray radiation must be eliminated or minimized. Recently, a method for reducing X-ray background signals from ID X-ray BPMs [4] has been implemented at two sectors (out of 40 sectors) at the APS storage ring. This method involves the introduction of a chicane into the accelerator lattice that directs unwanted background radiation away from the $\mathrm{X}$-ray BPM blades. The new X-BPM interface is designed to collect data from both the bending magnet and the insertion device beamlines, even though the initial plan is to implement the orbit feedback for all the bending magnet beamlines. The X-BPM orbit feedback study for the above-mentioned two insertion device beamlines will also proceed.

To study X-BPM orbit feedback characteristics in advance, an experimental set-up was developed using an existing X-BPM interface. This set-up was implemented in one of the bending magnet beamlines (BM14). Results from this set-up will also be discussed.

\section{BACKGROUND}

Before we could use X-ray BPMs for orbit feedback, we investigated whether the present X-ray BPM monitor system met the stringent submicron-level orbit readback requirements. An X-BPM interface requirement document [5] was developed that consists primarily of the following guidelines:

1. Provide blades and position data at $10 \mathrm{~Hz}$ to EPICS control system (to be used for monitoring and orbit feedback at DC level $<0.1 \mathrm{~Hz}$ ).

2. Provide position data up to $2 \mathrm{kHz}$ to the real-time orbit feedback system (to be used for monitoring and orbit feedback at AC level $-0.1 \mathrm{~Hz}$ to $30 \mathrm{~Hz}$ ).

3. Provide preamplifier gain control from EPICS.

4. Provide DSP software upgrade capability for ease of future development.

5. Provide controls for the translation stages from EPICS.

6. Provide dynamic range of $+/-1 \mathrm{~mm}$ with measurement resolution of 0.1 micron.

7. Provide stability performance of 0.1 micron in the frequency band of $1 \mathrm{~Hz}$ to $300 \mathrm{~Hz}$.

A detailed evaluation was done for the existing X-BPM interface and the results [6] revealed that the blade preamplifier noise floor and cross talk met the requirements. However, the data acquisition system did show several weaknesses, which resulted in its not meeting most of the requirements. An upgrade of the X-BPM data acquisition system is in progress and will be discussed in the next section. Even though all existing pre-amplifiers will be used, these units will be consolidated on a double sector basis to minimize data acquisition hardware. 


\section{SYSTEM DESCRIPTION}

The new system hardware design is in progress with a prototype unit scheduled to be tested during May and June, 2000. Figure 1 shows the block diagram. Three preamplifier chassis, each consisting of eight preamplifier units, are installed in one double sector rack. The signals from all blades will be routed to the back of these chassis using new cable installation. Even though the cable lengths are longer, tests have shown that there is no degradation in the signal-to-noise ratio. A 7-pole antialiasing filter module provides a $96 \mathrm{~dB}$ attenuation at $800 \mathrm{~Hz}$, which is half the present sampling rate. Since a large number of filter modules are required, an in-house design is used to lower cost. The present system has only one pole filter, which results in aliasing errors in the existing X-BPM orbit measurements.

A VMIC-3122 32-channel analog-to-digital converter has been tested that meets all the performance specifications [7]. This analog-to-digital converter can sample all channels up to $2 \mathrm{kHz}$. The gain control module provides an interface to all preamplifier units; each has seven gain ranges from which to select. The present interface system does not provide gain control via EPICS.

A dedicated DSP module (Pentek 4284) is used in the new X-BPM interface design. Some of its functions are:

1. Control and collect data from the analog-to-digital converter.

2. Control preamplifier gains via EPICS.

3. Compute position data at high speed and pass on to the real-time feedback DSP.

4. Filter blade and position data to $10 \mathrm{~Hz}$ and pass on to the workstation-based orbit feedback system. This data is also used for data logging.

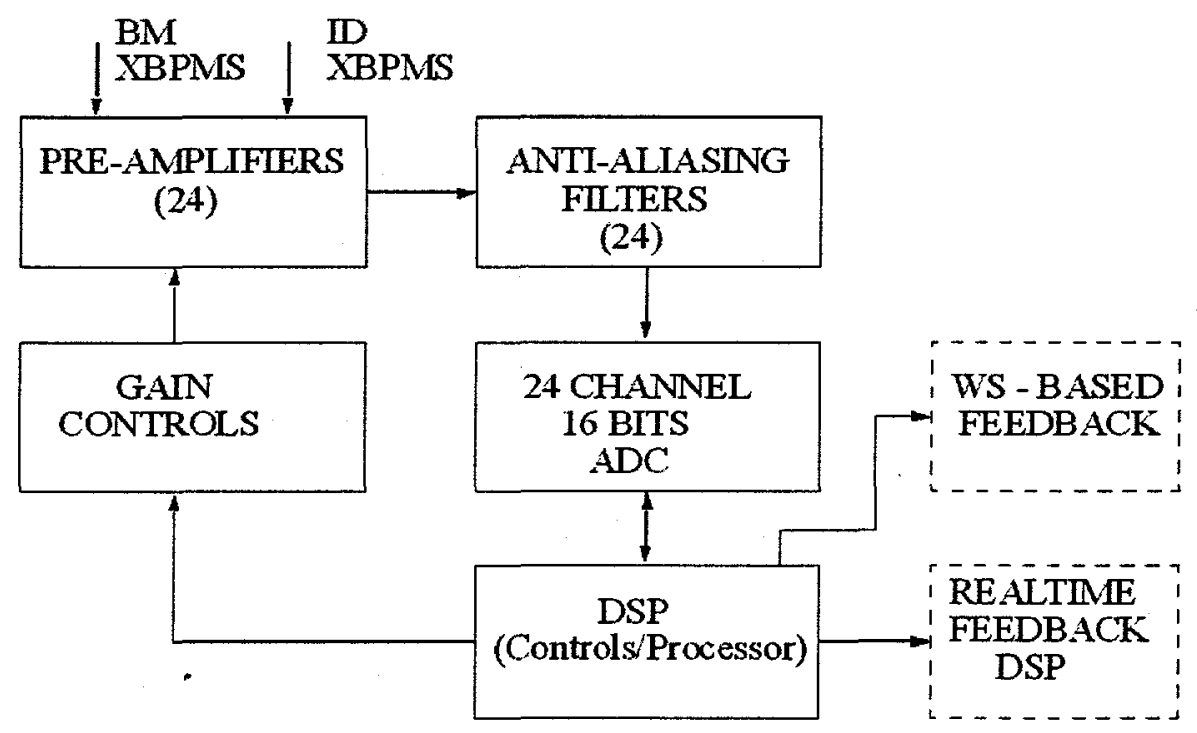

FIGURE 1. Block diagram of the new X-BPM interface. The dotted blocks show the existing rf BPM-based orbit feedback systems. 
The Pentek 4284 DSP was selected because a similar DSP is already in use for real-time feedback. No additional learning and development costs were involved as a result of this choice.

A prototype system is expected to be commissioned in sector $34 / 35$ where one of the sectors has the lattice modified for background signal reduction. The development of both the bending magnet and the insertion device X-BPM orbit feedback systems will progress together. In other sectors, bending magnet X-BPMs orbit feedback systems will be commissioned first as new interface systems become available.

\section{RESULTS FROM EXPERIMENTAL SET-UP}

An experimental set-up was tested in a bending magnet beamline (BM14) where $\mathrm{X}$-BPM data was made available to the workstation-based orbit feedback system. This set-up was achieved using the existing data acquisition system with four 6-pole antialiasing filters inserted at the output of each preamplifier. The existing software was also modified to provide $10-\mathrm{Hz}$ position data to the workstation-based orbit feedback system. Several changes were also made to the orbit control configuration, including 1) integrated responses of the X-BPMs into the rf BPM orbit correction configuration, 2) monitored the photon shutter, located between two X-BPMs, and 3) included several alarm conditions associated with X-BPM malfunctions.

Figure 2 shows the layout of the X-BPMs, bending magnets, and correctors used for orbit correction. For simplicity, if BPMs are not shown, but all "good" BPMs are included in orbit correction. Orbit correction at the APS employs a weighted singular value decomposition (SVD) algorithm, with the capability of using both $x$-ray and if BPMs. All if BPMs are assigned weights equal to "one" and X-BPMs, when used, are assigned weights equal to "five." With no X-BPMs included, only correctors a, d, and $\mathrm{f}$, as shown in Fig. 2, are used in this sector. When X-BPM(s) are included, three additional correctors $b, c$, and $e$ are utilized. The use of additional correctors ensures that the X-BPM's related correction is local to this sector.

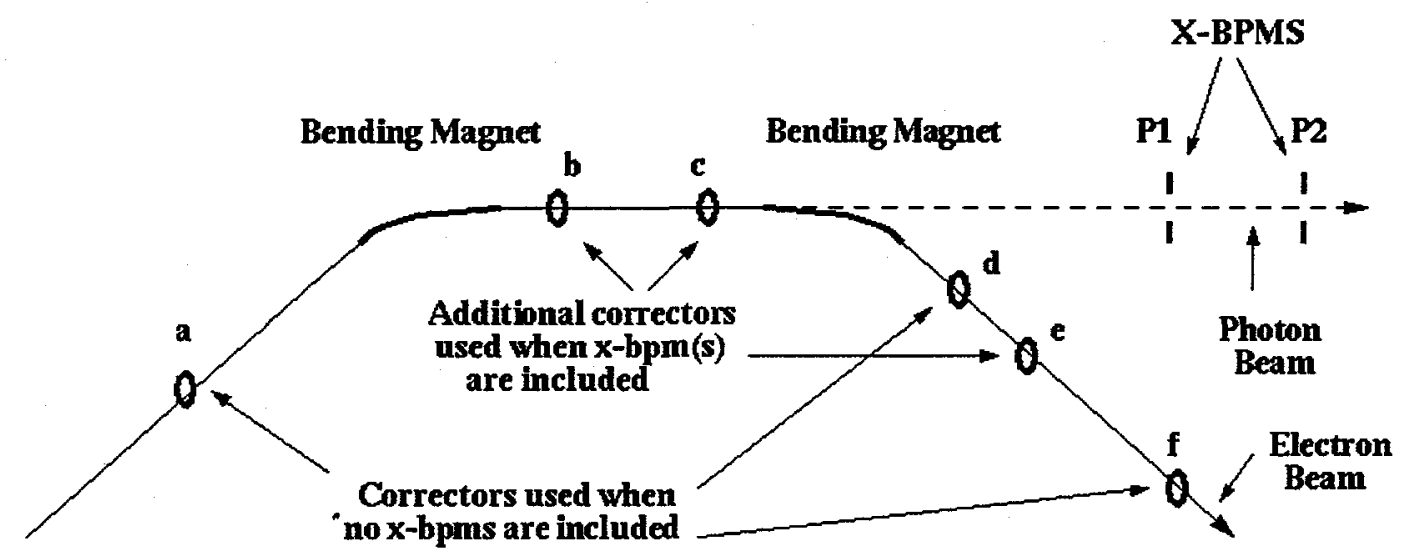

FIGURE 2. Layout of the $X$-ray BPMs $P 1$ and $P 2$, bending magnets, and six correctors $(a, b, c, d, e$ and f). For simplicity, rf BPMs are not shown. 
Figure 3 compares two operational BPM configurations - one as a standard configuration with no X-BPM added and the other with only one X-BPM (P1) added. The top traces show X-BPMs' drifts and beam current (DCCT) when no X-BPMs were included in the orbit configuration, and the bottom traces show X-BPM drifts and beam current (DCCT) when one X-BPM (P1) was included in the orbit configuration. The X-BPMs' drifts of 50 to 75 microns have been reduced to less than 10 microns due to the inclusion of one X-BPM. We chose to include only one XBPM, because we want to use other BPM as a check.

Figure 4 shows the corrector variations and storage ring beam current for an operational run with one X-BPM included for orbit correction. We noted that the dominant variations in the upstream three correctors $\mathrm{a}, \mathrm{b}$ and $\mathrm{c}$ exhibit what we will call a fill "thermal response," and the dominant variations in the downstream three correctors d, e and f exhibit what we will call an "intensity response." This is why we
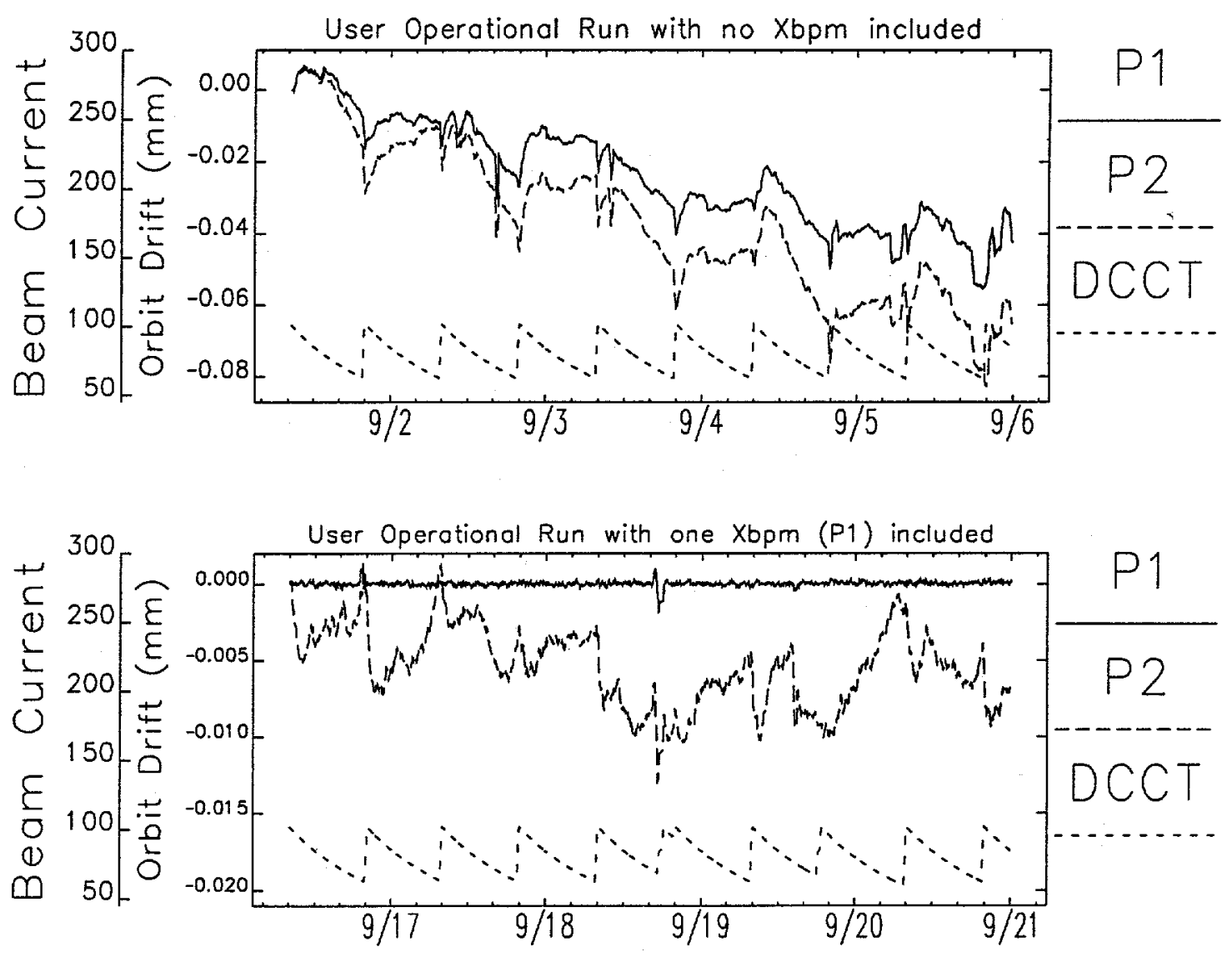

FIGURE 3. Comparison of orbit drift data with and without X-BPMs included in the DC orbit feedback. The X-BPM orbit drift of 75 microns over several operational fills (top traces) have been reduced to only 10 microns when one X-BPM (P1) was included in the orbit correction (bottom traces). 
have plotted the correctors in two groups. Each group of three correctors seem to form their own closed bump and suggest that the location of drift sources, i.e., the thermal type drift source, is upstream and the intensity type drift is downstream of the bending magnet. Further study will be done to investigate these effects.
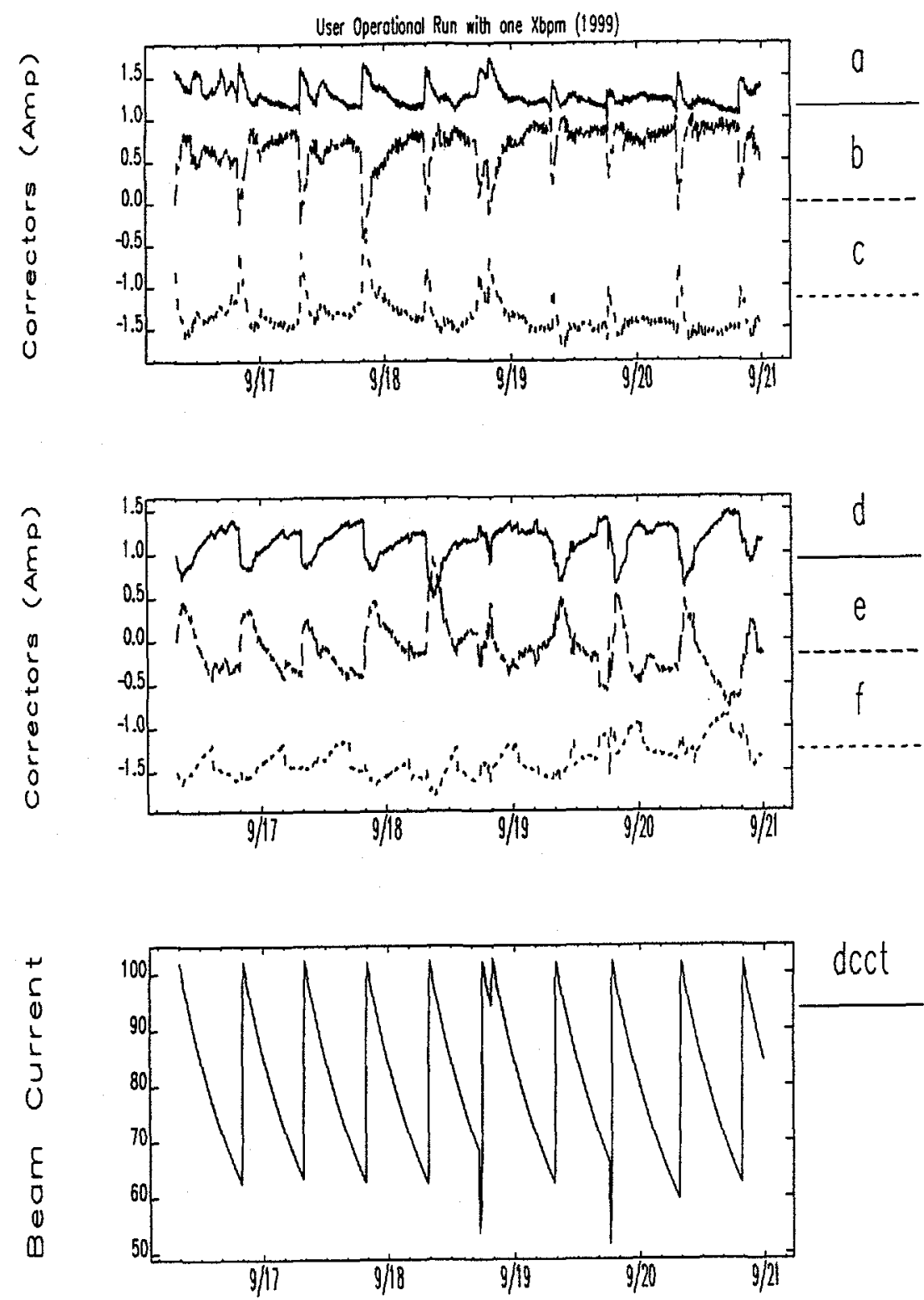

FIGURE 4. Storage ring beam current and corrector variation when one XBPM is included in the DC orbit feedback. The top trace shows three upstream correctors, the middle trace shows three downstream correctors, and the bottom trace shows the beam current. 


\section{CONCLUSIONS}

The results from the experimental set-up are encouraging. The X-BPM orbit drift has been reduced from 30 microns to less than 5 microns during one operational fill period of 12 hours, and from 75 microns to 10 microns over a $4 \frac{1}{2}$ day period.

A new X-BPM interface is under development that will allow integration of XBPMs into existing orbit feedback systems.

\section{ACKNOWLEDGMENTS}

We would like to acknowledge the efforts of Louis Emery (Operational Analysis Group), who modified several orbit correction configuration tools. This work is supported by the U.S. Department of Energy, Office of Basic Sciences, under Contract No. W-31-109-ENG-38.

\section{REFERENCES}

1. Shu, D., Barraza, J., Ding, H., Kuzay, T. M., and Ramanathan, M., "Progress of the APS High Heat Load X-ray Beam Position Monitor Development," in SRI: Tenth U.S. National Conference, 1997, pp. 173-177.

2. Emery, L., and Borland, M., "Advances in Orbit Correction in the Advanced Photon Source Storage Ring" in Proc. of the 1997 Particle Accelerator Conference, Vancouver, Canada, 1997, pp. 742-744.

3. Carwardine, J. A., and Lenkszus, F. R., "Real-Time Orbit Feedback at the APS," in $8^{\text {th }}$ Beam Instrumentation Workshop, edited by R. O. Hettel, S. R. Smith, and J. D. Masek, AIP Proceedings 451, Palo Alto, CA, 1998, pp. 125-144.

4. Decker, G., and Singh, O., Physical Review Special Topics - Accelerator and Beams 2, 112801 (1999).

5. Decker, G., private communication.

6. Lenkszus, F. R., private communication.

7. Lenkszus, F. R., private communication. 